\title{
Criminologie
}

\section{Le contrôle exercé sur la conjointe : comparaison de quatre groupes d'hommes}

\section{Benoît Roy et Gilles Rondeau}

Volume 30, numéro 2, automne 1997

Violences familiales

URI : https://id.erudit.org/iderudit/017404ar

DOI : https://doi.org/10.7202/017404ar

Aller au sommaire du numéro

\section{Éditeur(s)}

Les Presses de l'Université de Montréal

ISSN

0316-0041 (imprimé)

1492-1367 (numérique)

Découvrir la revue

Citer cet article

Roy, B. \& Rondeau, G. (1997). Le contrôle exercé sur la conjointe : comparaison de quatre groupes d'hommes. Criminologie, 30(2), 47-65.

https://doi.org/10.7202/017404ar
Résumé de l'article

This paper presents the results of an exploratory study on the control exerted by men over their spouse. Four groups of 15 men each, were compared: 1) men with no criminal record and who had volontarily taken part in a community program for violent men; 2) men accused of spouse abuse, exclusively; 3) men accused of spouse abuse and other crimes; 4 ) men selected at random from the community. The type of sample was non probabilistic. Three questionnaires were administered: 1) a demographic questionnaire 2) the "Abusive Behavior Inventory" (Shepard and Campbell, 1992) and 3) the "Psychological Maltreatment of Women Inventory" (Tolman, 1989). The results demonstrated that the control group (group 4) displayed "stability" in comparaison with the groups of accused men (groups 2 and 3) where important difficulties and "marginality" were observed. "Non accused" spouse abusers (group 1) obtained middle ranged scores. Scores from the four sub-scales in the two attitudinal inventories corrrelated significantly and were in the predicted direction. As the level of psychological or physical abuse increased, so did the desire to dominate and isolate women. Given the small sample size and the quasi-experimental nature of the design, the results are not considered as definitive; however, they provide directions for future research.
Ce document est protégé par la loi sur le droit d'auteur. L'utilisation des services d'Érudit (y compris la reproduction) est assujettie à sa politique d'utilisation que vous pouvez consulter en ligne.

https://apropos.erudit.org/fr/usagers/politique-dutilisation/ 
LE CONTRÔLE EXERCÉ SUR LA CONJOINTE : COMPARAISON DE QUATRE GROUPES D'HOMMES par Benoît Roy' et Gilles Rondeau²

This paper presents the results of an exploratory study on the control exerted by men over their spouse. Four groups of 15 men each, were compared: 1) men with no criminal record and who had volontarily taken part in a community program for violent men; 2) men accused of spouse abuse, exclusively; 3) men accused of spouse abuse and other crimes; 4) men selected at random from the community. The type of sample was non probabilistic. Three questionnaires were administered: 1) a demographic questionnaire 2) the "Abusive Behavior Inventory" (Shepard and Campbell, 1992) and 3) the "Psychological Maltreatment of Women Inventory" (Tolman, 1989). The results demonstrated that the control group (group 4) displayed "stability" in comparaison with the groups of accused men (groups $2 \& 3$ ) where important difficulties and "marginality" were observed. "Non accused" spouse abusers (group I) obtained middle ranged scores. Scores from the four subscales in the two attitudinal inventories corrrelated significantly and were in the predicted direction. As the level of psychological or physical abuse increased, so did the desire to dominate and isolate women. Given the small sample size and the quasiexperimental nature of the design, the results are not considered as definitive; however, they provide directions for future research.

\section{INTRODUCTION}

Cet article a pour but de présenter les résultats d'une recherche exploratoire portant sur le contrôle exercé par quatre groupes d'hommes sur leur conjointe. Dans le premier groupe, on retrouve des clients volontaires

1. M.Sc., travailleur social et assistant de recherche au Centre de recherche interdisciplinaire sur la violence familiale et la violence faite aux femmes (CRI-VIFF), Universite de Montréal, C.P. 6128, succursale Centre-Ville, Montréal, $Q c$, H3C 3J7. Adresse électronique : benroy@laurentides.net

2. Ph.D., professeurà l'École de service Social de l'Université de Montréal et chercheur au Centre de recherche interdisciplinaire sur la violence familiale et la violence faite aux femmes (CRI-VIFF) Université de Montréal, C.P. 6128, succursale Centre-Ville, Montréal, $\mathrm{Qc}, \mathrm{H} 3 \mathrm{C}$ 3J7. Adresse électronique : rondeaug@ere.umontreal.ca 
non judiciarisés inscrits à un programme pour conjoints violents, dans le deuxième groupe, des clients judiciarisés pour violence conjugale uniquement, et dans le troisième groupe, des clients judiciarisés pour violence conjugale et autres délits. Le quatrième groupe est un groupe témoin composé de conjoints non violents. Nous avons voulu savoir si les membres des divers groupes faisaient ou non usage de différentes formes de violence afin d'exercer un plus grand contrôle sur leur conjointe et si le degré de contrôle imposé augmentait selon la catégorie à laquelle ils appartenaient. Après avoir introduit la problématique de la violence conjugale et discuté de l'importance de la notion de « contrôle » qui s'y rattache, nous présenterons successivement la méthodologie de la recherche et les résultats obtenus.

\section{UNE PRÉOCCUPATION SOCIALE MAJEURE}

La violence conjugale est devenue une préoccupation majeure dans la société, suscitant l'intérêt de nombreux chercheurs et praticiens. Une étude récente de Statistique Canada (1993) brosse un sombre tableau de cette réalité définie comme « désignant les agressions physiques ou sexuelles qui correspondent aux définitions de ces infractions prévues par la loi et qui amèneraient la police à intervenir ». Malgré cette définition très restrictive, les résultats révèlent que $51 \%$ des femmes canadiennes disent avoir été victimes d'au moins un acte de violence physique ou sexuelle depuis qu'elles ont atteint l'âge de 16 ans. Vingt-cinq pourcent de l'ensemble des Canadiennes interrogées ont rapporté avoir subi, depuis l'âge de 16 ans, de la violence de la part de leur conjoint actuel ou précédent (Statistique Canada, 1993).

Au Québec, la politique en matière de violence conjugale présentée en 1986 par le ministre de la Justice suscita un large débat, obligeant les policiers à intervenir de façon active et considérant désormais les actes de violence entre époux au même titre que les autres formes d'agression. La violence sur la conjointe devint du coup répréhensible sur les plans social et juridique.

Les programmes de traitement pour les agresseurs sont très récents, les plus anciens remontant à 15 ans à peine. En 1988, Rondeau (1989) recensait 16 organismes œuvrant auprès des conjoints violents au Québec. À l'heure actuelle, l'Association des ressources intervenant auprès des hommes violents (ARIHV) comprend 26 organismes membres.

\section{CONCEPTS ASSOCIÉS À LA NOTION DE CONTRÔLE}

Lorsqu'on dépouilie la documentation sur la violence conjugale, on constate que les concepts utilisés demeurent encore relativement flous et imprécis. La notion de contrôle, par exemple, revêt des sens différents selon 
les auteurs. De même, des concepts voisins prendront des sens différents selon les textes, d'où l'importance de préciser ici leur signification.

\section{Autorité}

Le terme « autorité » ne réfêre pas à un acte particulier, mais d'abord à une fonction et à un rôle social (Marsal, 1966). En violence conjugale, il est fréquent que l'homme se perçoive comme investi d'une autorité, c'est-à-dire nanti d'un rôle social qui lui octroie une supériorité sur la femme. Selon Mendel (1971), avec l'autorité, il n'est nul besoin d'utiliser la force pour dominer :

L'autorité, masque mystifiant de la violence, permet d'obtenir par d'autres procédés que l'usage de la force nue, et donc avec une économie de dépenses, une attitude de soumission. Le supérieur domine, l'inférieur se soumet (p. 40).

\section{Pouvoir}

En violence conjugale, lorsqu'on parle de « pouvoir », on réfère généralement à un pouvoir de type coercitif (Hamilton, 1976). Celui-ci consiste à employer des sanctions négatives à l'endroit de la personne visée. On cherche ainsi à exercer un impact sur les autres, et on ira jusqu'à utiliser la force pour y parvenir si besoin est.

\section{Domination}

Le concept de « domination » réfère habituellement aux relations entre groupes, mais il peut néanmoins être appliqué aux relations interpersonnelles. Ce concept s'apparente à celui d'autorité en ce qu'il marque un rapport de déséquilibre entre deux groupes ou personnes alors que l'objectif du premier individu, le « dominant » est d'arriver à exercer une influence coercitive sur le second, le « dominé ».

\section{Violence psychologique}

Parce que moins apparente et plus polymorphe, la violence psychologique est plus difficile à cemer et à préciser que la violence physique. MacLeod (1987) a souligné l'importance de la première par rapport à la seconde :

Il est désormais clair et reconnu que la violence psychologique à laquelle sont soumises les femmes battues n'est pas une simple chicane de famille. La violence psychologique se manifeste généralement par des menaces ouvertes ou voilées ainsi que par une dégradation systématique de l'autonomie de la femme dans sa relation avec son partenaire, et souvent dans tous les aspects de sa vie (p. 15). 
La violence psychologique est une réalité complexe. Pour Kasian et Painter (1992), elle est fréquemment un signe précurseur de violence physique. Walker (1984, citée par Kasian et Painter, 1992) a identifié l'abus psychologique comme un comportement adopté par l'agresseur par lequel il vise à atteindre la victime afin de diminuer son estime de soi et son sentiment de sécurité et ainsi augmenter le contrôle qu'il peut exercer sur elle. Appleford (1989) définit ainsi la violence psychologique :

Par violence psychologique, on entend les menaces, le rejet chronique, l'absence d'intérêt, la critique, le confinement, l'isolement, l'humiliation, l'intimidation, la privation de droits, l'infantilisation, la destruction de biens ou un traitement, de la part d'un gardien ou d'un conjoint, visant à diminuer le sentiment d'identité, de dignité ou de valeur personnelle (p. 5).

Ouellet et al. (1996) ont effectué une étude sur les représentations que des hommes et des femmes aux prises avec la violence conjugale se faisaient de la violence psychologique. Leur démarche les a amenés à interroger 19 personnes (neuf femmes et dix hommes) sur les comportements qu'ils identifiaient comme relevant de la violence psychologique. Treize champs de référence furent rapportés, soit : le dénigrement, le contrôle, l'intimidation, le blâme et les accusations indues, la manipulation, la menace et le chantage, la privation intentionnelle, la surresponsabilisation/déresponsabilisation, la simulation et l'indifférence, la négation d'un état ou d'une condition, la bouderie, l'agression des enfants, le harcèlement.

Par ailleurs, un des outils utilisés dans la présente recherche est le Psychological Maltreatment of Women Inventory (Tolman, 1989). Il comprend deux sous-échelles, soit « domination et isolement » et « abus verbal et émotionnel ». Ces réalités constituent les deux grandes catégories sous lesquelles la plupart des comportements de violence psychologique peuvent être inclus. Lorsqu'on parle «d'abus verbal et émotionnel », par exemple, on identifie une façon d'agir, psychologiquement violente, qui inclut plusieurs formes. Il en est de même pour « domination et isolement ».

\section{Contrôle}

Les textes consultés ont permis de distinguer trois sens différents pouvant être attribués au concept de « contrôle ». Il y a d'abord la notion de contrôle prise dans son acception sociale. C'est dans ce sens qu'on parle du « contrôle social » comme d'une réalité sociale. Le second sens donné au terme est celui de «contrôle de soi ». Il va sans dire qu'en violence conjugale, ce n'est pas de ce type de « contrôle » dont on parle lorsqu'on mentionne que les conjoints violents exercent un contrôle. Cela étant, il y a cependant lieu de mentionner 
qu'à travers les objectifs de responsabilisation et de réappropriation poursuivis dans les programmes pour conjoints violents, on demande aux hommes de reconnaître qu'ils ont un contrôle sur eux-mêmes et qu'il leur appartient d'exercer ce contrôle de soi. Enfin, le troisième sens donné au terme " contrôle » renvoie essentiellement à l'exercice d'un rapport avec l'autre qui peut être défini comme l'actualisation d'une forme de coercition. C'est le sens du terme qui s'applique explicitement aux situations de violence conjugale.

Stets (1991) a compris la signification du terme de la façon suivante : «[...] le contrôle se définit comme l'action de diriger ou de tenter de diriger les pensées, les émotions et les actions d'une autre personne» (p. 101). Subotnik (1983) précise pour sa part que « [...] l'exercice du contrôle réfère à toute conduite qui a pour but d'exercer une influence sur l'autre personne ou de la dominer $\gg$ (p. 28).

Bailey (1983) a formulé une théorie selon laquelle plus quelqu'un possède de contrôle sur une personne, plus il est en mesure de l'atteindre jusque dans sa sphère intérieure. La façon d'atteindre cet intérieur chez la personne réside dans la connaissance la plus exacte possible des désirs, des sensations et des émotions de celle-ci, afin de la dominer. Ainsi, l'exercice du contrôle de l'homme sur sa conjointe sera facilité par la connaissance intime que l'agresseur a de sa victime.

Dans les situations de violence conjugale, le contrôle consiste essentiellement en l'exercice par une personne d'une action d'influence coercitive intentionnelle sur une autre personne avec qui une relation d'intimité est ou a été vécue à un moment ou un autre.

\section{UN ACTE DE DOMINATION ET DE CONTRÔLE}

L'idée qu'on se faisait de la violence conjugale s'est considérablement transformée depuis une quinzaine d'années. À l'origine, le terme « violence » référait essentiellement à des coups portés. Gelles et Strauss (1979), par exemple, souscrivaient à une définition de la violence qui se limitait aux blessures physiques.

Plus tard, la violence psychologique commença à être considérée plus attentivement, et on prit alors davantage conscience de ses effets. Il devint progressivement évident que la violence devait être redéfinie autrement, soit comme un acte de domination et de contrôle, un exercice de pouvoir habituellement exercé sur la conjointe.

Une telle forme de violence s'apparente davantage à un continuum. Plus que l'incident particulier, le contexte entier dans lequel la personne vit quotidiennement est perçu comme constituant la violence. 
Ils (les hommes violents) énoncent un continuum de violences et de contrôle où s'entrelacent différentes formes de violences, où en fonction de chaque moment ils choisissent une forme de violence approprié à l'état de la relation avec leur conjointe mais dont l'objectif et l'intention est d'imposer leur pouvoir et leur contrôle : contrôle de la situation et de leurs proches (Welzer-Lang, 1991, p. 199).

Faire cesser l'agression signifie alors davantage que de mettre un frein à la seule violence physique. Il faut tout aussi impérativement arrêter l'exercice de la violence psychologique. Le défi est de taille car, comme Ouellet et al. (1993) le rappelaient, " [...] la violence psychologique, notamment, est plus difficile à éliminer que la violence physique. » (p. 22) En effet, on touche là à tout le système de pensée et de valeur des conjoints violents eux-mêmes.

À la source des situations de violence conjugale, l'homme se définit comme investi d'une autorité, c'est-à-dire comme exerçant un rôle social qui lui octroie une supériorité sur la femme. L'usage de cette « autorité » lui permet d'obtenir par différents procédés autres que le simple usage de la force nue, et donc avec une économie de dépenses, une attitude de soumission (Mendel, 1971). À partir de la légitimation de son autorité, le conjoint utilisera un «pouvoir » coercitif en faisant un usage bien défini de la menace, de la force ou des deux conjointement (Tedeshi et al., 1977) afin d'atteindre la soumission désirée. Un rapport « dominant-dominé » sera ainsi établi sur le plan des relations interpersonnelles dans le couple. Afin de maintenir ce rapport, la violence psychologique deviendra le moyen couramment employé. Au besoin, l'agresseur recourra à la violence physique. L'ensemble du processus peut être vu sous l'angle de l'imposition d'un contrôle défini comme l'actualisation d'une forme de coercition où l'abuseur dirige ou tente de diriger les pensées, les émotions et les actions d'une autre personne, sa conjointe (Stets, 1991).

La tactique de contrôle coercitif n'a d'autre but que de maintenir le pouvoir de l'agresseur sur la personne visée (Yllö, 1993, citée par Hydén, 1995). Gondolf (1988b) appuie cette affirmation en soutenant que les comportements abusifs du mari à l'endroit de sa femme sont faits dans une perspective de maintien ou de gain de pouvoir et de contrôle sur celle-ci et s'inscrivent dans une société de type patriarcal. La portée du contrôle imposé dépasse bien évidemment la situation immédiate où sont impliqués deux partenaires. Selon Kaufman (1987), « les structures de domination et les formes de contrôle ne sont pas simplement à l'arrière-plan des formes de violence mais engendrent et éduquent ce phénomène de violence» (p. 12).

Plusieurs chercheurs ( Tedeshi et al., 1977 ; Allen et Straus, 1980; Kaufman, 1987 ; Stets, 1988 : Claes et Rosenthal, 1990 ; Subotnik, 1990 ; 
Wartenberg, 1990 ; Berkowitz, 1993 ; Petrik et al., 1994 ; Prince et Arias, 1994) ont tenté de trouver l'origine et de mieux comprendre la dynamique de domination, de contrôle et de déséquilibre du pouvoir existant au sein du couple dans un contexte de violence conjugale.

Selon la théorie féministe explicative de la violence conjugale, le désir ou le besoin de contrôle de l'homme sur sa conjointe prend sa source dans le système patriarcal, où la femme est vue comme subordonnée à l'homme qui détient la supériorité. Un tel contexte référentiel amène l'homme à croire a la légitimité de son autorité. Dans ce cadre, l'utilisation de la violence, quelle que soit sa forme, sera d'abord perçue moins comme un abus que comme l'utilisation de la force nécessaire pour établir, maintenir ou restaurer l'autorité " légitime ». Seuls les excès seront condamnés, à savoir lorsque, de façon patente, des moyens inutilement brutaux seront utilisés.

C'est ce genre d'éthique qui prévaut lorsqu' on applique ce principe aux rapports hommes-femmes dans la société patriarcale. La légitimation de l'autorité entraînant celle du contrôle, il importe qu'en ce qui concerne les pays et sociétés, l'homme et la femme soient proclamés et reconnus libres et égaux afin de supprimer toute prétention de légitimité découlant d'une position d'autorité.

Malgré cette reconnaissance d'égalité, plusieurs hommes continuent à se considérer en droit d'employer des modes de coercition diversifiés pour marquer leur autorité et leur possession sur leur conjointe, exerçant un contrôle plus ou moins entier sur celle-ci.

Cela étant, nous allons maintenant voir comment, concrètement, différents hommes exercent ou pas ce contrôle et se comparent entre eux sous ce rapport.

\section{QUESTION DE RECHERCHE}

Cette recherche emploie un devis quantitatif-descriptif et vise, dans une perspective exploratoire, à vérifier l'existence de relations quantitatives entre différentes variables sélectionnées. Son objectif principal est de mieux connaître et, par le fait même d'arriver à mieux comprendre, les facteurs associés à l'exercice du contrôle de l'homme sur sa conjointe en contexte de relation conjugale violente. Les résultats obtenus à ce premier stade exploratoire ont pour objectif d'arriver éventuellement à la formulation d'une ou de plusieurs hypothèses devant être testées dans des recherches ultérieures.

À partir des articles recensés et des expériences de travail des auteurs, il fut supposé que le degré de contrôle des hommes sur leurs conjointes était susceptible de varier selon le degré de violence physique ou psychologique 
exprimé dans le passé. Ainsi, les hommes condamnés pour violence conjugale et autres délits furent présumés plus contrôlants que ceux qui avaient été condamnés pour violence conjugale uniquement. Ces derniers furent à leur tour présumés plus contrôlants que ceux qui se reconnaissaient violents mais qui n'avaient jamais été judiciarisés. Enfin, ceux-ci furent présumés plus contrôlants avec leurs conjointes que les hommes « ordinaires » choisis dans la société et constituant le groupe témoin. Ce qui fut donc présumé est un comportement contrôlant descendant en quantité et qualité, allant du plus élevé au plus faible, selon le degré correspondant de violence démontrée dans le passé.

\section{MÉTHODOLOGIE}

\section{Sujets}

Pour mener à bien la vérification de la question, l'échantillon a été subdivisé en quatre catégories de répondants : 1) hommes violents judiciarisés pour violence conjugale et pour d'autres types d'infractions en vertu du code criminel ; 2) hommes violents judiciarisés uniquement pour violence conjugale ; 3 ) hommes violents non judiciarisés et en début de traitement pour violence envers la conjointe, et 4) hommes ne présentant pas de comportement déclarés de violence et n'ayant pas de dossier criminel (groupe témoin).

Le type d'échantillon retenu est non probabiliste. Au total, la participation de 15 hommes par groupe a été visée afin d'obtenir un nombre suffisant de sujets pour atteindre les objectifs de mesure. La sélection a respecté les critères suivants : tous les participants devaient être des résidents de la région des Laurentides âgés de 18 à 65 ans. Les hommes constituant le groupe témoin ont été recrutés au CLSC Arthur-Buies lorsqu'ils s'y sont présentés pour des entrevues sans rendez-vous. Ceux qui ont constitué le groupe des hommes violents non judiciarisés et en début de traitement pour violence cnvers lcur conjointe ont été choisis parmi les nouveaux participants d'ACCROC de StJérôme, un organisme communautaire dispensant des services aux conjoints violents. Les hommes violents judiciarisés ont été sélectionnés à partir des dossiers ouverts au Service de probation provincial de la région des Laurentides et au Centre de détention provincial de Saint-Jérôme.

La sélection s'est faite au hasard en ce sens que dans chaque catégorie, tous les hommes qui satisfaisaient aux critères étaient abordés pour participer à l'étude, jusqu'à ce que le quota de 15 ait été atteint. La sélection des participants et la distribution des questionnaires se sont étendues sur une période de six mois, de janvier à juin 1994. Toutes les entrevues ont été 
menées avec l'assentiment des participants. Aucun avantage, quel qu'il soit, n'était lié à la participation à l'étude. Chacun des 60 participants a été rencontré individuellement par le premier auteur, qui leur a alors fait remplir les questionnaires en situation de face-à-face.

\section{Instruments de mesure utilisés}

Trois questionnaires ont ainsi été administrés à l'ensemble des sujets. Le premier visait à recueillir un ensemble de données psychosociales sur les participants. Élaboré par le premier auteur, il présentait les informations selon une échelle nominale. Les données psychosociales recueillies portaient sur les six derniers mois.

Les deux autres questionnaires furent l'Abusive Behavior Inventory (ABI), de Shepard et Campbell (1992) et le Psychological Maltreatment of Women Inventory (PMWI), de Tolman (1989). Il s'agit de deux échelles d'attitude qui présentent les données de façon ordinale. Chacune des échelles est construite pour obtenir les réponses selon le système gradué en cinq points développé par Likert. Les deux questionnaires d'attitudes ont été traduits de l'anglais par une traductrice professionnelle. La procédure classique de « traduction et retraduction » n'a pu être réalisée. Toutefois, un test de vérification de la traduction a été effectué auprès de deux groupes de personnes différentes extérieures au projet.

Un ensemble de tests statistiques a été conduit, tenant compte du petit nombre de sujets compris dans les groupes et de la fréquence des réponses aux différentes parties des questionnaires. Ainsi, à la suite de l'analyse des fréquences, une analyse de variance a été effectuée afin de déterminer si les quatre sous-groupes de l'échantillon se différenciaient au niveau des variables « revenu » et « scolarité ». Par la suite, les résultats des sous-groupes aux quatre sous-échelles prévues ont été compilés et comparés. Une analyse de corrélation entre les sous-échelles d'attitude a enfin été effectuée afin de vérifier la force de leurs liens.

L'instrument, l'Abusive Behavior Inventory (ABI), consiste en une échelle d'attitude développée par les chercheurs américains Shepard et Campbell en 1992. Il s'agit d'un questionnaire de 30 items divisé en deux sous-échelles, l'une mesurant l'abus psychologique et l'autre l'abus physique. La sous-échelle sur l'abus psychologique est constituée de vingt items et se subdivise en six catégories thématiques : 1) abus émotionnel : humiliation et dégradation, 2) isolement : restriction des contacts sociaux, 3) intimidation : craintes ou peurs suscitées par des actions ou des gestes, 4) menace : attaque dirigée contre la conjointe ou des substituts, 5) abus financier : restriction des ressources financières, 6) utilisation des privilèges liés aux stéréotypes 
sexuels masculins. La sous-échelle d'abus physique comporte pour sa part dix items et ne comprend pas de sous-catégories.

Le second instrument, le Psychological Maltreatment of Women Inventory ( $P M W I)$, est une échelle d'attitude créée par Richard M. Tolman (1989). Composée de 58 items, l'échelle a été adaptée à partir d'instruments existants. L'auteur s'est notamment inspiré du Conflict Tactic Scale, de Straus (1979) pour cinq items et de l'Index of Spouse Abuse, de Hudson et MacIntosh (1981) pour 16 items. L'échelle d'attitude se divise en deux sous-échelles, soit 1) domination et isolement, comprenant 26 items et 2) abus émotionnel et verbal, 23 items. Les neuf items restants peuvent être attribués à l'une ou l'autre des sous-échelles, sans distinction. Les deux outils emploient un système gradué de réponses en cinq points allant de « aucun abus $=0$ » à « abus fréquent $=5$ ». Le calcul du score de chaque sous-échelle s'obtient en additionnant chacune des réponses et en divisant par le nombre d'items inclus dans l'unité de mesure. Il n'y a de score global pour aucune des deux échelles, seules les sous-échelles ayant été validées ${ }^{3}$.

La décision de faire passer les échelles d'attitude à ces différents groupes a été prise pour pouvoir d'abord vérifier si les différentes formes de violence utilisées variaient selon le type d'homme impliqué. En deuxième lieu, il y avait la volonté de comparer une population d'agresseurs judiciarisés à une population d'agresseurs non judiciarisés afin de vérifier dans quelle mesure les uns se distinguaient des autres.

3. L'Abusive Behavior Inventory (ABI), a fait l'objet, par ses auteurs, des tests de validation suivants : l'outil a été testé sur un échantillon de près de 200 personnes. L'erreur standard de mesure (standard error of measurement) se distribue de .04 a .12 , avec un coefficient Alpha de fidélité de 70 à .92 . La validité de critères décrite par les auteurs donne un résultat adéquat : abus psychologique ( $r=.48$ version homme et $r=.50$ version femme) et abus physique ( $r=.49$ version homme ct $r=.38$ version femme). Enfin, lá sous-échelle d'abus physique a un coefficient Alpha de .80 à .92 , et la sous-échelle d'abus psychologique un coefficient Alpha de .76 a .91. Pour sa part, le Psychological Maltreatment of Women Inventory ( $P M W I)$ a été testé et validé par l'auteur. L'analyse de facteurs pour les deux souséchelles réalisée par l'auteur est adéquate tant pour la version homme $(r=.7320)$ que pour la version fermme ( $r=.7376$ ). Le Rho de Spearmean's révèle une corrélation significative ( $r=$ $.4849, p=.7376$ ) pour la sous-échelle domination et isolement dans ces deux versions. Pour la sous-échelle de l'abus verbal et émotionnel, il n'y a pas de corrélation significative ( $r=$ $.3025, p=.265$ ). Le coefficient de validité inteme entre les sous-échelles de la version homme est important (domination/isolement, $\infty=0.9087$; abus émotionnel et verbal, $\infty=0.9335$ ). Les deux instruments comprennent chacun une version pour l'homme agresseur et une version pour la femme victime. Les chercheurs ne disposaient malheureusement pas de moyens leur permettant de solliciter l'opinion de la conjointe, ce qui aurait permis une meilleure vérification des réponses des participants. 


\section{Limites}

Les limites de la présente recherche tiennent d'abord à son échantillon qui est non probabiliste. La procédure de sélection suivie ne permet pas de généraliser les résultats à l'ensemble de la population étudiée. Le nombre restreint de sujets de l'échantillon constitue par ailleurs une seconde limite à la portée des résultats. Enfin, le fait que les deux principaux instruments de mesure aient été traduits directement de l'américain, sans validation de la qualité de cette traduction, est susceptible d'affaiblir la valeur des résultats obtenus.

\section{RÉSULTATS OBTENUS}

\section{Données sociodémographiques}

Les données sociodémographiques recueillies ont permis de tracer un profil propre à chaque sous-groupe de participants. La comparaison fait ressortir des points intéressants. On remarque que le groupe témoin est le seul sous-groupe qui comprend des hommes ayant poursuivi des études universitaires $(33 \%)$. La proportion d'assistés sociaux est demeurée identique entre les trois groupes de conjoints violents $(26,7 \%)$, se situant très légèrement au-dessus de celle du groupe témoin $(20 \%)$. De façon surprenante, c'est dans le groupe d'hommes violents judiciarisés pour violence conjugale et pour d'autres types d'infractions qu'on trouve le plus de personnes travaillant à plein temps, soit $(60 \%)$. Alors que cette proportion n'est que de $53.3 \%$ parmi le groupe contrôle, $40 \%$ parmi le groupe de conjoints non-judiciarisés et en début de traitement, et $33.3 \%$ parmi le groupe de conjoints judiciarisés pour violence conjugale seulement. Il s'agit toutefois d'emplois mal rémunérés qui se comparent mal avec ceux du groupe témoin. Les revenus vont en diminuant à partir du groupe témoin. Celui-ci jouit d'un revenu beaucoup plus élevé que les autres groupes. Il est suivi par les conjoints violents non judiciarisés et en début de traitement. Les participants à ce groupe se séparent en deux sous-groupes de taille égale, soit les participants qui vivent dans la pauvreté avec un revenu annuel inférieur à $10000 \$$ et ceux qui gagnent plus de $35000 \$$. Les hommes violents judiciarisés uniquement pour violence conjugale sont dans des tranches de revenu moins polarisées mais ont globalement une moyenne inférieure. Enfin, les hommes violents judiciarisés pour violence conjugale et autres délits sont ceux dont le revenu moyen est le plus faible de tous.

On aura noté ici que, de façon générale, les membres du groupe témoin jouissent d'un niveau de scolarité et d'un revenu plus élevés que ceux des trois groupes de conjoints violents. Cette différence peut être imputée au fait que le groupe témoin a été constitué à partir de la population générale de la région des Laurentides s'adressant au CLSC Arthur-Buies. La population de 
la région des Laurentides jouit d'un niveau socioéconomique supérieur à la moyenne provinciale, alors que les groupes de conjoints violents sont formés, au moins au deux tiers, de personnes judiciarisées, soit un segment de la population reconnu comme sous-scolarisé et plus pauvre que la moyenne. Ces différences sociodémographiques dans la composition des groupes soulevaient des questions sur la validité des résultats. Nous avons conséquemment voulu vérifier si nos résultats demeuraient significatifs lorsque nous contrôlions le niveau de scolarité et le revenu ${ }^{4}$.

Dans les deux groupes de conjoints violents judiciarisés, on note un très fort pourcentage d'hommes vivant en union de fait $(86,7 \%)$ comparativement aux deux autres groupes ( 53 et $50 \%$ ). La durée des relations conjugales semble suivre une courbe d'allure similaire à celle des revenus, à savoir que le groupe témoin est celui où il y a le plus de relations actuelles longues (quatre ans et plus) et les groupes d'hommes violents judiciarisés ceux où il y en a le moins. Les conjoints violents non judiciarisés et en début de traitement se situent entre les deux mais sont paradoxalement ceux qui connaissent le plus haut taux de séparations et de divorces. Une très forte proportion de conjoints violents $(86,7 \%)$ ont déjà fait vie commune avec une autre femme que leur compagne actuelle. Si plus des deux tiers des répondants $(68,3 \%)$ vivent avec des enfants, on observe des différences entre les sous-groupes sous ce rapport. Ainsi, les hommes du groupe témoin vivent tous avec des enfants alors qu'à l'opposé, le sous-groupe judiciarisé pour violence conjugale comprend la plus faible proportion $(33,3 \%)$ de répondants vivant avec des enfants. Dans les deux autres sous-groupes, un pourcentage relativement élevé habite avec des enfants $(40,0 \%$ pour le sous-groupe en traitement et $60,0 \%$ pour le sous-groupe judiciarisé pour violence conjugale et autres délits).

À la différence des hommes du groupe témoin, les conjoints violents mentionnent en majorité avoir été témoins ou victimes de violence dans leur famille d'origine. Les deux groupes de conjoints judiciarisés comprennent quelques hommes ayant reçu les services d'un psychiatre, ce qui est exceptionnel dans les deux autres groupes. Le pourcentage du sous-groupe d'hommes judiciarisés pour violence conjugale uniquement ayant reçu les services d'un psychiatre $(33,3 \%)$ est plus important que celui de l'autre sousgroupe judiciarisé $(26,7 \%)$. Dans un autre ordre d'idées, il est intéressant de noter que plus du tiers des hommes formant les deux sous-groupes judiciarisés avaient déjà participé à un programme de traitement pour agresseurs conjugaux. Il importe également de souligner qu'une très forte proportion des hommes violents non judiciarisés et en début de traitement pour violence envers la conjointe $(73,3 \%)$ n'en étaient pas à leur première

4. Voir plus loin dans la section « Résultats aux échelles d'attitude ». 
participation à ce genre de programme thérapeutique. Enfin, en ce qui a trait à l'alcool, la tendance à boire avec excès est presque inexistante chez les participants du groupe témoin, alors qu'elle grimpe a $20 \%$ chez les conjoints violents non judiciarisés et à $33 \%$ chez les deux groupes judiciarisés.

Globalement, on peut dire qu'il ressort une image de stabilité chez les participants du groupe témoin alors que ceux des deux groupes judiciarisés illustrent davantage des difficultés importantes et une marginalisation sociale. Le groupe des conjoints violents non judiciarisés se situe entre les deux. On y note la présence de tensions, mais aussi une moins grande désorganisation et une recherche de stabilité plus marquée.

\section{Résultats aux échelles d'attitude}

L'analyse de la variance effectuées pour les deux sous-échelles de l'Abusive Behavior Inventory (Shepard et Campbell, 1992) révèle que le groupe témoin se distingue de façon significative ${ }^{6}$ des deux sous-groupes judiciarisés. Ainsi, à la sous-échelle « abus psychologique », le score moyen du groupe témoin $(1,37)$ est significativement plus faible que celui du groupe des répondants judiciarisés pour violence conjugale $(2,03)$ et celui du groupe des répondants judiciarisés pour violence conjugale et autres infractions criminelles $(2,14)$. Le score moyen du groupe des répondants non judiciarisés et en début de traitement $(1,78)$ ne présente pas une différence statistiquement significative par rapport aux scores des autres groupes pour la sous-échelle de l'abus psychologique.

Pour la sous-échelle d' « abus physique », les résultats de l'analyse de variance font état d'une distinction significative entre, d'une part, les deux sous-groupes judiciarisés $(1,49$ et 1,52$)$ et, d'autre part, le groupe témoin et le groupe des répondants non judiciarisés et en début de traitement $(1,07$ et 1,15).

En ce qui a trait au Psychological Maltreatment of Women Inventory (Tolman, 1989), les résultats des répondants nous indiquent, par l'analyse de la variance, que le groupe témoin $(34,17$ et 41,50$)$ se distingue de façon significative des deux sous-groupes judiciarisés $(51,30$ et 57,$53 ; 52,81$ et $61,38)$ aux deux sous-échelles, soit respectivement « domination et isolement " et « abus verbal et émotionnel ». Les scores du groupe non judiciarisé en début de traitement aux mêmes deux sous-échelles, $(42,88$ et 52,36) sont inférieurs à ceux des conjoints judiciarisés pour violence conjugale seulement $(51,30$ et 57,53$)$ et à ceux des conjoints judiciarisés pour violence conjugale et autres délits $(52,81$ et 61,38$)$, tout en étant supérieurs à ceux du groupe témoin $(34,17$ et 41,50$)$.

5. Test de Turkey.

6. $\mathrm{P} \leq 0,05$. 
L'intercorrélation des résultats entre les quatre sous-échelles des deux échelles d'attitude nous indique que les corrélations (coefficient de Pearson) entre les sous-échelles sont statistiquement significatives. Les résultats aux quatre sous-échelles ont des totaux de plus en plus élevés à partir du groupe témoin jusqu'au groupe des hommes judiciarisés pour violence conjugale et autres délits. Les sous-échelles « abus psychologique » et « dominationisolement » obtiennent le résultat d'intercorrélation le plus fort (.9244), ce qui concorde avec le cadre théorique. On note également que la corrélation entre les sous-échelles «domination-isolement " et « abus verbal et émotionnel » (.6883) se situe près de celle observée entre les sous-échelles « abus physique » et « abus verbal et émotionnel ». Tout en demeurant significatives, les corrélations « abus psychologique »-« abus physique » (.7322) ; « abus psychologique »-« abus verbal et émotionnel » (.7184); « abus physique »-« domination et isolement » (.7151) ont des coefficients assez semblables. Une explication à ces résultats vient peut-être des redondances existant entre différentes questions des sous-échelles. Se peutil qu'indistinctement des termes employés, on évalue finalement des réalités relativement semblables lorsqu'on mesure l'abus psychologique, l'abus verbal et émotionnel ou la recherche de domination et d'isolement ?

Les résultats aux deux sous-échelles de l'Abusive Behavior Inventory indiquent une polarisation entre le groupe témoin d'un côté et les trois sousgroupes d'agresseurs de l'autre. Le score du groupe témoin à la sous-échelle de l'abus psychologique est statistiquement différent des deux sous-groupes d'agresseurs judiciarisés. Les résultats aux deux sous-échelles du Psychological Maltreatment of Women Inventory témoignent aussi d'une certaine polarisation entre, d'un côté, le groupe témoin, et, de l'autre, les deux sous-groupes d'agresseurs judiciarisés. Les résultats du sous-groupe de conjoints violents en début de traitement sont supérieurs à ceux du groupe témoin, sans que cette différence s'avère significative.

Le niveau de scolarité et de revenu du groupe témoin est supérieur à celui des trois autres groupes. Nous avons voulu savoir si certaines des différences observées pouvaient être attribuées aux écarts entre les membres du groupe témoin et ceux des autres groupes relativement à ces variables. Pour ce faire, nous avons cherché à contrôler le niveau de scolarité et de revenu. Comme il s'agit de variables dichotomiques, nous avons fait une analyse de variance par strates ${ }^{7}$. Pour chacun des scores aux quatre sous-

7. En raison du petit nombre de sujets, nous avons regroupé le niveau d'éducation en deux catégories, soit $1=$ niveau primaire et secondaire et $2=$ niveau collégial et universitaire. De même, nous avons regroupé le revenu en deux catégories : $1=$ moins de $20000 \$$ et $2=20000 \$$ et plus. 
échelles, nous avons ainsi stratifié pour le niveau de scolarité d'une part et le revenu d'autre part. Le test d'interaction indique une interaction non significative pour les quatre scores, qu'on considère le niveau de scolarité ou le revenu ${ }^{8}$. On peut donc dire que les différences observées entre les quatre groupes sont du même ordre, quel que soit le revenu ou le niveau de scolarité. Cela étant, comme le nombre de sujets est petit, il pourrait être intéressant et informatif de considérer les moyennes des quatre groupes selon les deux niveaux de revenus ou de scolarité, et de dégager les tendances ${ }^{9}$. On peut dire, malgré les lacunes méthodologiques relevées, que les tendances observées dans le cadre de cette recherche sont suffisamment fortes pour justifier la mise en place d'un projet semblable de plus grande envergure. Celui-ci devra alors inclure un échantillon de plus grande taille et de type probabiliste.

Dans un autre ordre d'idées, en mettant en corrélation les quatre souséchelles, on constate que plus l'abus psychologique et l'abus physique sont importants chez l'agresseur, plus la volonté de dominer et d'isoler la conjointe sont élevés. On note, d'autre part, que l'abus psychologique de l'agresseur est lié de façon significative à l'abus verbal et émotionnel. L'abus physique est lié, de façon significative, à la domination de l'autre et au fait d'isoler la conjointe des autres personnes. Les liens entre abus physique et abus verbal et ceux entre domination-isolement et abus verbal et émotionnel, bien que plus faibles, demeurent tout de même significatifs.

8. Résultats au test d'interaction :

Sous-échelle « Abus psychologique »

Sous-échelle « Abus physique »

Sous-échelle « Domination et isolement »

Sous-échelle « Abus verbal et émotionnel »

$\begin{array}{cc}\text { Revenu } & \text { Scolarité } \\ .309 & .707 \\ .654 & .868 \\ .487 & .238 \\ .250 & .572\end{array}$

9. L'espace nous manque pour inclure ici les tableaux de moyennes des scores des quatre groupes à chacune des échelles selon les deux catégories de revenu et les deux catégories de niveau de scolarité. L'analyse de ceux-ci nous a permis de dégager les tendances suivantes. On observe au niveau du revenu que les scores augmentent par paliers successifs du groupe témoin au groupe des conjoints non judiciarisés à celui des conjoints judiciarisés pour violence conjugale seulement et enfin à celui des hommes judiciarisés pour violence conjugale et autres crimes, et ce, pour les quatre sous-échelles. Cette tendance se maintient dans les deux catégories de revenu, exception faite du groupe des hommes judiciarisés pour violence conjugale et autres délits, qui ont des revenus inférieurs. De façon constante, ceuxci ont un score moyen légèrement inférieur à celui du groupe des hommes judiciarisés pour violence conjugale seulement aux quatre sous-échelles. Sur le plan de la scolarité, la tendance notée est de même nature. Ici aussi, les scores augmentent par palier à partir du groupe témoin jusqu'au groupe des hommes judiciarisés pour violence conjugale et autres délits pour ce qui est des hommes ayant un faible niveau de scolarité. Pour ceux qui ont une scolarité plus élevée, les scores fluctuent davantage entre les catégories, mais celles-ci comprennent très peu de sujets. 
Les résultats obtenus soulignent l'existence d'un lien entre l'exercice du contrôle, l'utilisation de la violence et la judiciarisation des agresseurs. On observe ainsi des résultats croissants en terme de comportements contrôlants chez nos quatre sous-groupes allant du groupe témoin à celui des hommes judiciarisés pour violence conjugale et autres délits. Plus la criminalisation des agresseurs est lourde, plus les manifestations de violence sont sévères. Ainsi, l'homme davantage violent et judiciarisé sera porté à utiliser un plus grand éventail de formes de violence et à exercer un contrôle plus étendu sur sa conjointe, cherchant à la dominer au niveau de ses paroles, de ses actions et essayant de s'immiscer jusque dans ses pensées.

La recherche de la domination et de l'isolement de la conjointe constitue une des composantes de l'abus psychologique. L'étude des corrélations entre les résultats aux quatre sous-échelles amène à faire le constat que les abus de type verbal-émotionnel ou de type domination-isolement ne s'accompagnent pas forcément d'abus physiques. La violence physique peut être peu employée si elle n'est pas indispensable pour assurer la domination sur la conjointe. Il n'en reste pas moins que le degré de violence psychologique imposé peut être très élevé et persistant.

\section{CONCLUSION}

En agissant de façon physiquement ou psychologiquement violente ou en menaçant de le faire, l'agresseur cherche à influencer sa conjointe afin qu'elle se conforme à ses désirs ou besoins. Tel que souligné dans la première partie de l'article, il apparaît que l'agresseur cherche confirmation de son jugement personnel a priori, à savoir que l'homme est supérieur à la femme et a ainsi autorité sur elle et les enfants. Pour imposer son autorité, l'homme estime légitime d'utiliser un pouvoir coercitif sur sa conjointe et d'exercer le contróle par divers moyens.

L'utilisation des deux échelles d'attitude a permis de mesurer avec plus d'acuité la volonté de contrôle des conjoints violents et d'identifier, sur le plan du comportement, le degré de contrôle effectivement exercé par ces hommes sur leurs conjointes. Les questionnaires ont aussi permis de noter de façon plus précise comment s'actualisait l'exercice du contrôle dans une relation conjugale violente.

À partir de la documentation consultée et des résultats de cette recherche exploratoire auprès de 60 hommes, il y a lieu d'être préoccupé des intentions des agresseurs relativement à l'utilisation de la violence comme moyen de contrôle de la conjointe. Ceux-ci semblent effectivement retirer des gains significatifs de leur recours à la violence pour instaurer et maintenir le contrôle sur leur partenaire. 
Il importe qu'une recherche similaire, avec un échantillon plus grand et de type probabiliste, soit entreprise afin de vérifier si les tendances observées dans la présente recherche exploratoire se vérifient. Il faut en effet aller plus avant sur la piste afin de démontrer ou d'infirmer de façon rigoureuse l'existence des liens observés.

Sur un autre plan, évaluer le parcours suivi par l'agresseur pour arriver à exercer le contrôle apparaît comme une des étapes à franchir pour mieux cerner le phénomène. De même, le développement de meilleurs instruments d'évaluation de la violence psychologique semble s'imposer. Ceux-ci sont nécessaires pour effectuer une évaluation plus poussée et judicieuse de cette dimension. Ces deux mesures combinées faciliteraient l'élaboration d'une intervention thérapeutique davantage adaptée aux diverses réalités de la violence conjugale. Dans cette perspective, la poursuite de la recherche et de la réflexion constituent une nécessité parce qu'elles permettront d'arriver à une connaissance plus exacte de l'agresseur et, partant, d'améliorer l'intervention tant dans sa forme thérapeutique que sociale.

\section{BIBLIOGRAPHIE}

ALLEN, M. C. et STRAUS, A. M. (1980), Ressources, Power and Husband-Wife Violence, in Straus A. M. et Hotaling T. G., The Social Causes of Husband-Wife Violence, (p. 188208), Minneapolis : University of Minnesota Press.

APPLEFORD ASSOCIATES (1989), Étude documentaire sur la violence familiale: Prévention et traitement, Ottawa : Service Correctionnel Canada.

BERKOWITZ, L. (1993), Agression: Its Causes, Consequences, and Control, USA : McGraw-Hill inc.

CLAES, A. J. et ROSENTHAL, M. D. (1990), Men Who Batter Women : A Study in Power, Journal of Family Violence, 5, (3), 215-224.

GELLES, R. J. et STRAUS, M. A. (1979), Determinants of Violence in the Family : Towards a Theoretical Integration, in W. Burr et al., (eds), Contemporary Theories About the Family, New York : Free Press.

GONDOLF, E. W. (1988a), Research on Men who Batter. An Overview, Bibliography and Resource Guide, Bradenton, Florida : Human Services Institute.

GONDOLF, E. W. (1988b), Who Are Those Guys? Towards a Behavioral Typology of Batterers, Violence and Victims, 3, (3), 187-203.

HAMBERGER, L. K. (1994), Domestic partner abuse : Expanding Paradigms for Understanding and Intervention, Violence and Victims, 19, (2), 91-94.

HAMBERGER, L. K. et HASTINGS, J. E. (1986), Personality Correlates of Men Who Abuse Their Partners : A Cross-Validation Study, Violence and Victims, 1, (4), 323341.

HAMBERGER, L. K. et HASTINGS, J. E. (1988), Recidivism Following Spouse Abuse Abatement Counseling: Treatment Program Implications, 20 Atlanta GA : Paper presented at the Meeting of the American Psychological Association. 
HAMBERGER, L. K. et HASTINGS, J. E. (1991), Personality Correlates of Men Who Batter and Nonviolent Men : Some Continuities and Discontinuities, Joumal of Family Violence, 6, (2), 131-147.

HAMILTON, M. (1976), An Analysis and Typology of Social Power (part 1), Philosophy of the Social Sciences, 7, 51-65.

HOLTZWORTH-MUNROE, A. et STUART, G. L. (1994), Typologies of Male Batterers : Three Subtypes and the Differences Among Them, Psychological Bulletin, 116, (3), 476-497.

HUDSON, W. W. et MCINTOSH, R. S. (1981), The Assessment of Spouse Abuse : Two Quantifiable Dimensions, Journal of Marriage and the Family, 873-888.

HYDÉN, M. (1995), Verbal Agression as Prehistory of Woman Battering, Journal of Family Violence, 10, (I), 55-71.

KASIAN, M. et PAINTER, L. S. (1992), Frequency and Severity of Psychological Abuse in a Dating Population, Journal of Interpersonnal Violence, 7, (3), 350-364.

KAUFMAN, M. (1987), The Construction of Masculinity and the Trial of Men's Violence, in Kaufrnan, M. (ed) Beyond Patriarchy : Essays by Men on Pleasure, Power and Change, Toronto/New York : Oxford University Press, 1-29.

MACLEOD, L. (1987), Pour de vrais amours... Prévenir la violence conjugale, Ottawa : Conseil Consultatif Canadien sur la situation de la femme.

MARSAL, M. (1966), L'autorité, Paris : Presses Universitaines de France, Coll. Que saisje?

MENDEL, G. (1971), La violence, Paris, Presses Universitaires de France, Coll. Que saisje ?

OUELLET, F., LINDSAY, J. et SAINT-JACQUES, M.-C. (1993), Évaluation de l'efficacité d'un programme de traitement pour conjoints violents, Université Laval : Centre de recherche sur les services communautaires, 263 pages.

OUELLET, F., LINDSAY, J., CLÉMENT, M. et BEAUDOIN, G. (1996), La violence psychologique entre conjoints, Tomes I et 2 CRI-VIFF, Montréal et Québec, 169 pages.

PETRIK, N., OLSON-PETRIK, E. R. et SUBOTNIK, S. L. (1994), Powerlessness and the Need to Control : The Male Abuser's Dilemna, Journal of Interpersonal Violence, 9, (2), 272-285.

PRINCE, E. J. et ARIAS, I. (1994), The role of Perceived Control and the Desirability of Control Among abusive and Nonabusive husbands, The American Journal of Family Therapy, 22, (2), 126-134.

RONDEAU, G. (1989), Les programmes québécois d'aide aux conjoints violents : Rapport sur les seize organismes existants au Québec, Québec: Directions générale de la planification et de l'évaluation, ministère de la Santé et des Services sociaux.

RONDEAU, G. (1995), Violences masculines : faits et choix, Actes du Ir Congrès mondial sur la violence et la coexistence humaine, Montréal, Éditions Montmorency, vol. III, 269-286.

ROY, B. (1995), L'exercice du contrôle par l'agresseur dans une relation conjugale violente, Mémoire de maîtrise en service social, non publié, Université de Montréal.

SAUNDERS, D. G. (1987), Are There Different Types of Men Who Batter? An Empirical Study with Possible implications for Treatment, Durtam : University of New-Hampshire, Paper presented at the Third National Conference for Family Violence Researchers. 
SHEPARD, F. M. et CAMPBELL, A. J., (1992), The Abusive Behavior Inventory : A Measure of Psychological and Physical Abuse, Joumal of Interpersonnal Violence, 7, (3), 291305.

STATISTIQUE CANADA (1993), L'enquête sur la violence envers les femmes, Le Quotidien, 18 novembre 1993 , p. 1-10.

STETS, E. J. (1988), Domestic Violence and Control, New York : Spinger-Verla.

STETS, E. J. (1991), Psychological Agressions in Dating Relationships : The Role of Interpersonnal Control, Journal of Family Violence, 6, (1), 97-113.

STRAUS, M.A. (1979), Measuring Intrafamily Conflict and Violence : The Conflicts Tactics Scales, Journal of Marriage and the Family, 41, February, 75-88.

SUBOTNIK, L. S. (1990), Overcontrolled and Undercontrolled Types of Men Who Batter Women, Mémoire de mâ̂trise, non publié, University Northern Colorado.

TEDESHI, T. J., GAES, G. G. et RIVERA, N. A. (1977), Agression and the Use of Coercive Power, Journal of Social Issues, 33, (1), 101-125.

TOLMAN, M. R. (1989), The Development of a Measure of Psychological Maltreatment of Women by their Male Partners, Violence and Victims, 4, (3), 159-177.

TOLMAN, R. M. et BENNET, L. W. (1990), A Review of Quantitative Research on Men who Batter, Journal of Interpersonal Violence, 5, (1), 87-118.

WALKER, L. E. (1979), The Batterred Women, New York : Harper and Row.

WARTENBERG, E. T. (1990), The Forms of Power : From Domination to Transformation, Philadelphia : Temple University Press.

WELZER-LANG, D. (1991), Les hommes violents, Paris, Lierre et Coudrier Éditeur.

YLLÖ, K. (1984), The Status of Women, Marital Equality, and Violence Against Wives, Journal of Family Issues, 5, (3), 307-320. 